Supporting Information

\title{
INOSINE NUCLEOBASE ACTS AS GUANINE IN INTERACTIONS WITH PROTEIN SIDE CHAINS
}

Matea Hajnic, Anita de Ruiter ${ }^{\#}$, Anton A. Polyansky and Bojan Zagrovic ${ }^{*}$

Department of Structural and Computational Biology, Max F. Perutz Laboratories, University of Vienna, Campus Vienna Biocenter 5, Vienna, A-1030, Austria

\#present address: Anita de Ruiter, Institute of Molecular Modeling and Simulation, BOKU, Muthgasse 18, Vienna, A-1190, Austria

*o whom correspondence should be addressed. Tel: +43 14277 52271; Fax: +43 14277 9522; Email: bojan.zagrovic@univie.ac.at 

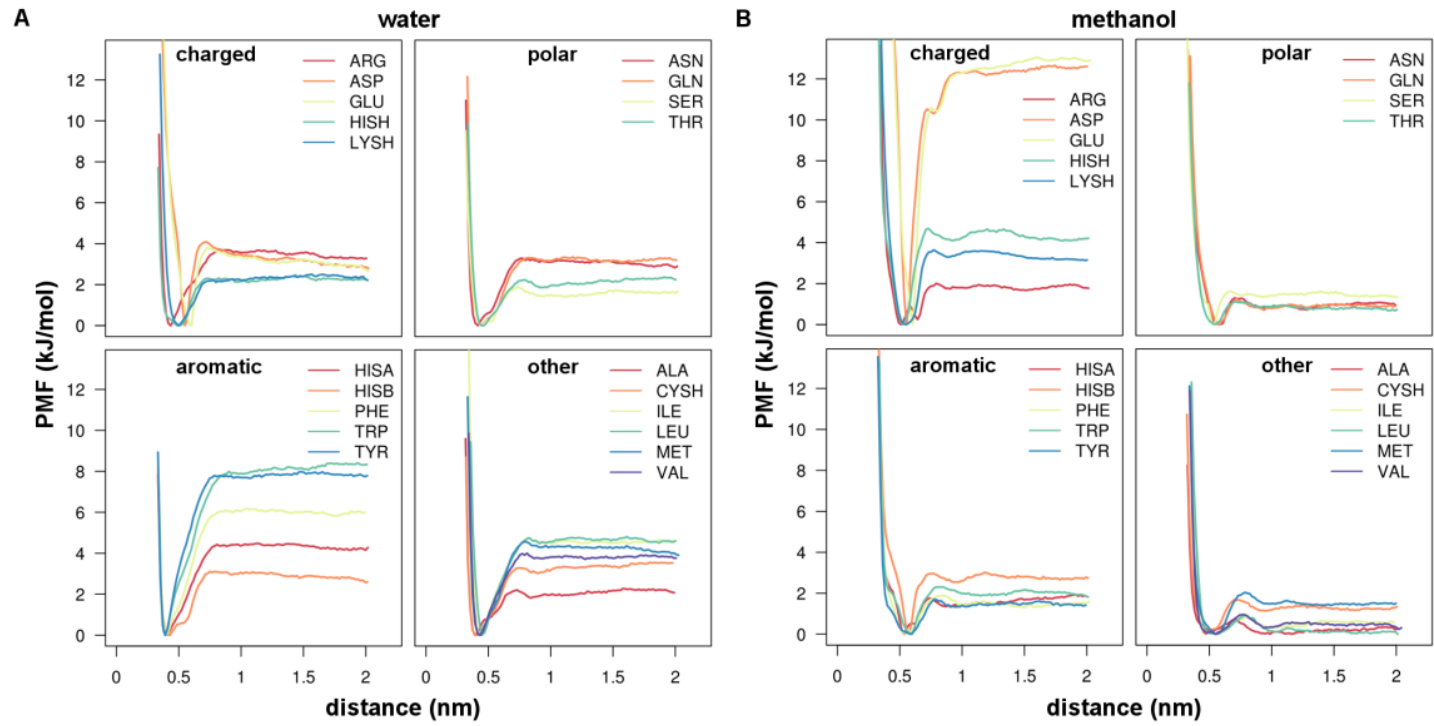

Figure S1. Potential of mean force (PMF) curves for the binding between hypoxanthine and different amino acid side chain analogs derived in $\mathbf{A}$ ) water and $\mathbf{B}$ ) methanol. 


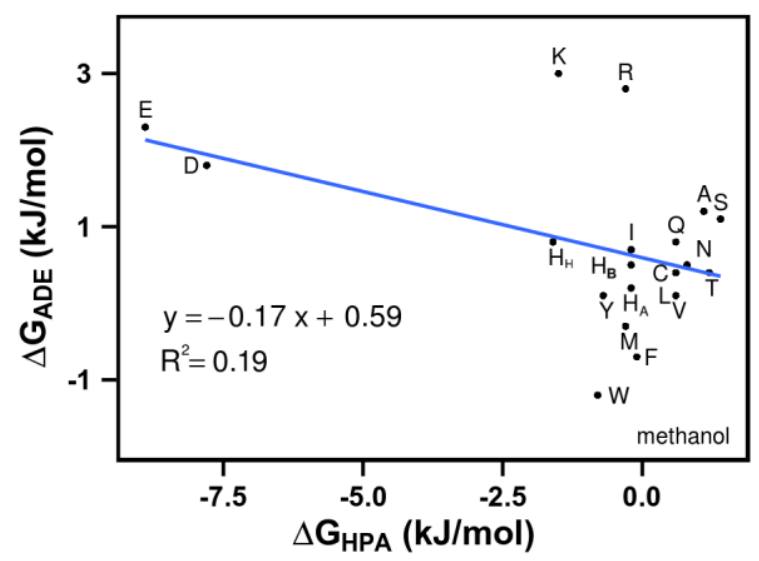

Figure S2. Correlation between the binding free energies with amino acid side chain analogs of hypoxanthine (HPA) and adenine (ADE) ${ }^{1}$ derived in methanol. 
Table S1. Pearson $\mathrm{R}^{2}$ coefficients of the binding-free energies derived between amino acid side chain analogs and HPA/nonmodified ${ }^{1}$ nucleobases in $\mathbf{A}$ ) water and $\mathbf{B}$ ) methanol.

A)

\begin{tabular}{lcccccc} 
Water & & & & & & \\
& HPA & GUA & ADE & CYT & URA & THY \\
\hline HPA & 1 & 0.94 & 0.81 & 0.90 & 0.85 & 0.77
\end{tabular}

B)

Methanol

\begin{tabular}{ccccccc} 
& HPA & GUA & ADE & CYT & URA & THY \\
\hline HPA & 1 & 0.89 & 0.19 & 0.66 & 0.34 & 0.40
\end{tabular}


Table S2. Root mean square deviations (RMSD) in $\mathrm{kJ} / \mathrm{mol}$ between the binding-free energies derived between amino acid side chain analogs and HPA/nonmodified ${ }^{1}$ nucleobases in $\mathbf{A}$ ) water and $\mathbf{B}$ ) methanol.

A)

Water

\begin{tabular}{ccccccc} 
& HPA & GUA & ADE & CYT & URA & THY \\
\hline HPA & 0 & 0.52 & 0.69 & 0.97 & 0.89 & 1.31
\end{tabular}

B)

Methanol

\begin{tabular}{ccccccc} 
& HPA & GUA & ADE & CYT & URA & THY \\
\hline HPA & 0 & 1.14 & 3.57 & 2.26 & 3.76 & 4.42
\end{tabular}




\section{Comparison of Plasmodium and human HGPRT}

We have also compared the affinities of the human HGPRT enzyme for hypoxanthine to its homolog from Plasmodium falciparum, the causative agent of malaria. HGPRT from Plasmodium is more promiscuous as in addition to catalyzing the conversion of hypoxanthine and guanine to their nucleotide forms, it catalyzes the same reaction for xanthine. Since HGPRT variants from humans and Plasmodium differ in specificity, the enzyme has received attention as a potential drug target. Specifically, recent work using Raman spectroscopy and these two HGPRT homologs suggested that the basis of Plasmodium promiscuity with respect to the human enzyme lies in how tight the substrate is bound in the transition state $^{2}$. We have used the available crystal structures of the Plasmodium HGPRT enzyme in the substrate-bound state and the transition state, and have compared the estimated affinities of the binding pockets for hypoxanthine between the two homologous enzymes. We did not observe any difference in the affinities for hypoxanthine between the two enzymes in the substrate-bound state $\left(\Delta \mathrm{G}_{\text {Plasmodium }}=-5.45\right.$ $\mathrm{kJ} / \mathrm{mol}, \Delta \mathrm{G}_{\text {human }}=-5.66 \mathrm{~kJ} / \mathrm{mol}$ ), while in the transition state the human enzyme binds hypoxanthine more favorably $\left(\Delta \mathrm{G}_{\text {Plasmodium }}=-5.45 \mathrm{~kJ} / \mathrm{mol}, \Delta \mathrm{G}_{\text {human }}=-6.95 \mathrm{~kJ} / \mathrm{mol}\right)$ supporting the proposal ${ }^{10}$ that the promiscuity of Plasmodium HGPRT relative to that in human may partly be due to the difference in tightness of the bound nucleobase in the transition state.

\section{METHODS}

\section{Molecular dynamics (MD) simulations}

We have used MD simulations in combination with umbrella sampling to derive the binding-free energies of 18 natural amino acid side chain analogs (all except for Gly and Pro) and hypoxanthine (HPA) in water and methanol. In all simulations in water, a single amino acid side chain analog and a hypoxanthine nucleobase were placed in a cubic simulation box (with the length of a cubic box between $4.5 \mathrm{~nm}$ and $4.9 \mathrm{~nm}$ ) with their centers of geometries set at $2 \mathrm{~nm}$ apart with no preferential conformation. All simulations were carried out using the GROMACS 5.0. simulation package ${ }^{4}$, united-atom GROMOS $54 \mathrm{a} 8^{2}$ force field and the SPC water model (with 3074 to 3878 molecules per box, depending on the system) with a 2 fs integration step. Ionizable sidechain analogs were 
set to correspond to a $\mathrm{pH}$ of 7. The parameters for hypoxanthine were derived from those corresponding to the most similar nucleotides in the GROMOS $54 \mathrm{a} 8$ force field ${ }^{2}$. The adenine nucleobase parameters were used as a scaffold for hypoxanthine, where only the amino group at position C6 had to be changed to carbonyl oxygen (parameters for the given chemical group were taken from guanine nucleobase). In addition, methyl group at position N9 was added, to the place where nucleobase gets attached to sugar via glycosidic bond. Sidechain analogs were parameterized to match the corresponding amino acids with a backbone part replaced by a methyl group. All bonds were constrained using LINCS $^{5}$. Non-bonded interactions within $0.8 \mathrm{~nm}$ range were calculated based on a pairlist that was updated every 5 steps. The interactions between 0.8 and 1.4 nm were calculated only with every pairlist update and were kept constant otherwise. Interactions beyond $1.4 \mathrm{~nm}$ were accounted for by a reaction field contribution with a dielectric permittivity of 61 , which is appropriate for SPC water ${ }^{6}$. The temperature and pressure were kept at $298 \mathrm{~K}$ and $1 \mathrm{~atm}$ throughout, using Berendsen thermostat ${ }^{7}\left(\tau_{\mathrm{T}}=0.1\right.$ ps) and Berendsen barostat $\left(\tau_{\mathrm{p}}=0.5 \mathrm{ps} \text { and compressibility }=4.5 \times 10^{-5} \mathrm{bar}^{-1}\right)^{7,8}$, respectively. All settings, except for the following ones, were kept the same for the simulations in methanol. First, the size of the simulation boxes stayed the same as in water simulations, but with the number of solvent molecules between 1366 and 1765 , depending on the system. The pressure was again kept at 1 atm using the same barostat as for simulations in water, but with a different compressibility $\left(1.25 \times 10^{-4} \mathrm{bar}^{-1}\right)^{9,10}$. In addition, non-bonded interactions beyond the $1.4 \mathrm{~nm}$ cutoff were accounted for by a reaction field contribution with a dielectric permittivity of $18.6^{11}$.

After minimization using the steepest descent algorithm in water and methanol (25000 steps), the system was equilibrated in six independent steps. In the first step, positions restraints were applied (with a force constant of $2.5 \times 10^{4} \mathrm{~kJ} \mathrm{~mol}^{-1}$ ) to solute molecules, while the initial velocities were drawn from the Maxwell-Boltzmann distribution at $50 \mathrm{~K}$. In the next four equilibration steps, the temperature was raised each time by $50 \mathrm{~K}$ and the force constant of the position restraints lowered by a factor of 10 . In the last equilibration step, the temperature was set to $298 \mathrm{~K}$, while position restraints were switched-off and 
center-of-mass-translation removed every 1000 steps. The first four equilibration steps were simulated for 20 ps each, while the last step took 40 ps.

\section{Umbrella sampling}

The distance between the centers of geometry of hypoxanthine and different amino acid side chain analogs was used as the reaction coordinate for the derivation of potentials of mean force. Umbrella sampling was used to enhance the sampling along the reaction coordinate by applying harmonic distance restraints with a force constant of $500 \mathrm{~kJ} \mathrm{~mol}^{-1}$ $\mathrm{nm}^{-2}$ as a biasing potential. The restraining distances used in simulations ranged from 0.4 $\mathrm{nm}$ to $1.9 \mathrm{~nm}$ separated by steps of $0.1 \mathrm{~nm}$, that in total resulted in 16 independent simulations runs for each side chain/hypoxanthine pair. At each step, we first performed an equilibration of $100 \mathrm{ps}$ that was followed by production run of $10 \mathrm{~ns}$. To test for convergence, we split the production runs into 5-ns long segments, and for each of them calculated the PMFs from which the binding free energies were determined. If the difference in binding free energies determined from the first and the second 5-ns segments was greater than $1.5 \mathrm{~kJ} / \mathrm{mol}$, the production runs for all distances were prolonged for additional $10 \mathrm{~ns}$ until this criterion was met. The relative rotational correlation times of Trp/hypoxanthine, the pair with the strongest affinity and involving the largest amino-side chain analog, in water equal 10 ps, 10 ps and 710 ps at the distances where their centers of geometries (COG) are $1.9 \mathrm{~nm}, 1.1 \mathrm{~nm}$ and $0.4 \mathrm{~nm}$ apart, respectively. In methanol simulations, the relative rotational correlation times for the same side chain/nucleobase pair equal 12 ps, 13 ps and 98 ps at the COG distances of 1.9 $\mathrm{nm}, 1.1 \mathrm{~nm}$ and $0.4 \mathrm{~nm}$, respectively. This suggests that our simulation time of $10 \mathrm{~ns}$ per umbrella point is adequate for proper sampling. The binding free energies were calculated from the PMF curves using the methodology previously described in de Ruiter et $\mathrm{al}^{1}$.

\section{Analysis of spatially-averaged affinities in the HGPRT binding site}

The spatially-averaged affinities of HGPRT protein side chains reported in the manuscript (Figure 2) were calculated in the following way. For each residue in question, a sphere with a radius of $7 \AA$ was positioned at its $\mathrm{C}_{\text {alpha }}$ atom and the nucleobase affinities, derived in our methanol simulations, of all residues with their $\mathrm{C}_{\text {alpha }}$ atoms 
within the sphere were summed up and normalized by the total number of residues within the sphere. We have also evaluated the spatially-averaged affinities for sphere radii of 5 $\AA$ and $6 \AA$ and the final results did not differ qualitatively from those reported in the manuscript for any of the five key residues in the enzyme binding site except for Phe 186 at the radius of $5 \AA$ where the affinities are the same for all four catalytic stages. 


\section{REFERENCES}

(1) de Ruiter, A.; Zagrovic, B. Nucleic Acids Res. 2014, 43 (2), 708.

(2) Karnawat, V.; Gogia, S.; Balaram, H.; Puranik, M. ChemPhysChem 2015, 16 (10), 2172.

(3) Pronk, S.; Páll, S.; Schulz, R.; Larsson, P.; Bjelkmar, P.; Apostolov, R.; Shirts, M. R.; Smith, J. C.; Kasson, P. M.; van der Spoel, D.; Hess, B.; Lindahl, E. Bioinforma. Oxf. Engl. 2013, 29 (7), 845.

(4) Reif, M. M.; Hünenberger, P. H.; Oostenbrink, C. J. Chem. Theory Comput. 2012, 8 (10), 3705.

(5) Hess, B.; Bekker, H.; Berendsen, H. J. C.; Fraaije, J. G. E. M. J. Comput. Chem. 1997, 18 (12), 1463.

(6) Heinz, T. N.; van Gunsteren, W. F.; Hünenberger, P. H. J. Chem. Phys. 2001, 115 (3), 1125.

(7) Berendsen, H. J. C.; Postma, J. P. M.; van Gunsteren, W. F.; DiNola, A.; Haak, J. R. J. Chem. Phys. 1984, 81 (8), 3684.

(8) Haynes, W. M. CRC Handbook of Chemistry and Physics, 94th Edition, 94th ed.; 2013.

(9) Marcus, Y. Wiley: The Properties of Solvents - Yizhak Marcus, P.G.T. (ed),; John Wiley \& Sons, Ltd, Chisester, 1998.

(10) Caleman, C.; van Maaren, P. J.; Hong, M.; Hub, J. S.; Costa, L. T.; van der Spoel, D. J. Chem. Theory Comput. 2012, 8 (1), 61.

(11) Walser, R.; Mark, A. E.; van Gunsteren, W. F.; Lauterbach, M.; Wipff, G. J. Chem. Phys. 2000, 112 (23), 10450. 